\title{
SIMULATION: STUDY OF FLOW OF GAS IN A RESISTIVE PLATE CHAMBER
}

\author{
S.D.Kalmani ${ }^{1}$, P.Verma ${ }^{2}$, P.V.Hunagund ${ }^{3}$ \\ ${ }^{1,2}$ Tata Institute of Fundamental Research, Homi Bhaba Road, Mumbai- 400005 \\ ${ }^{3}$ Gulbarga University Campuses, Applied Electronics Department Gulbarga- 585106 \\ kalmani@tifr.res.in
}

\begin{abstract}
The RPC (Resistive Plate Chamber) detectors are widely used in many large scale based mega science experiment s like CMS, ATLAS etc. This detector will also be used as an active element in the INO (India Based Neutrino Observatory) experiment and will have about $30 K$ RPC detectors. The RPC with glass electrode is considered for the studies. The constituent elements of RPCs are two parallel electrode, made of high resistivity float glass, between which, in a $2 \mathrm{~mm}$ gap, a mixture of gas viz. $R 134 a\left(C_{2} H_{2} F_{4}\right.$ $: 1,1,1,2$ tetraflouro ethane) , $i C_{2} H_{4}$ (Isobutane) and $S_{6}$ (sulphur hexafluoride) in the ratio of 95::4.5::0.5 respectively, is circulated. Earlier studies have indicated that, the concentration and quantity of gas mix plays a very important role in the performance of the RPCs. The current drawn by the chambers rises if the amount of pollutants in the mixture increases and the performance deteriorates. . It is important to estimate progress of partial pressure gradient of a component of gas mixture or a radical generated, over a period of time of transit during process of detection. Therefore, a number of studies on controlling and optimisation of the gas flow through the RPCs is required.

In this paper we are presenting the simulation results related to the (a) Transient flow pattern of gas inside the RPC detector say form 0 second to 60 seconds using Freon (R22) gas (major component of a mixed gas). (b) Gas flow inside the RPC detector for small flow rate few SCCM (velocity from 0.01meters /second to 0.18 metres/second), as the glass RPC are very efficient in low flow rate when the outside environment of operation has low radiation (underground INO experiment, while in CMS it is quite high due to collusion of photons and antiprotons).

Simulation is done on a platform of COMSOL Multiphysics® version 5.1 in 2 dimensions, to study the flow velocity of gas within a RPC gap whose dimensions are 1.8 meter X 1.9 meter with a gap of $2 \mathrm{~mm}$ and assuming $3 \mathrm{~mm}$ as thick glass, that could be used in the INO experiment.

Each gas of the mixture has different property such as density, viscosity and diffusivity at operating temperature and therefore a concentration gradient will setup within the gas mixture. The segregation of gases under different velocity conditions may occur.
\end{abstract}

KEYWORDS: RPC, Gaseous Detector; Calorimeter, CFD, Gas Simulation.

\section{INTRODUCTION :}

INO (India Based Neutrino Observatory), a large mega science project funded by Department of Science and Technology and Department of Atomic energy, Govt. of India., will use RPC (Resistive Plate Chamber) as one of active component for construction of $50 \mathrm{~K}$ ton magnetised Iron Calorimeter detector (ICAL) [1][2][3]. The "coated glass gap" with a gap of $2 \mathrm{~mm}$ created in between two $3 \mathrm{~mm}$ thick float glass with four gas nozzles, and these glasses are coated with conductive paint on the outer surface, while the surfaces inside the gap are cleaned and not coated. The $2 \mathrm{~mm}$ gap is maintained by button spacers placed at distances of $200 \mathrm{~mm}$ both along $\mathrm{X}$ and $\mathrm{Y}$ directions and by side spacers of thickness of $2 \mathrm{~mm}$, on the four edges. So a gap of $2 \mathrm{~mm}$ is created inside the chamber and the buttons and side spacer are glued with $3 \mathrm{M}$ glue. There are two gas inlets and two outlets for gas flow as shown in the Figure 1A.

The proposed INO-ICAL detector will have about 30,000 RPCs. The details of the design can be found in [1][2]. The

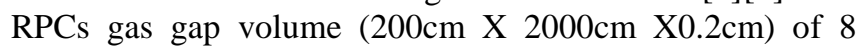
litres is filled with a gas mixture which is primary signal producing media of the detector. The total volume of the mixed gas in the ICAL would be about $240 \mathrm{~m}^{3}$. The gas mixture also decides the mode of operation of the detectors namely the avalanche mode (Freon, Isobutane, Sulphur hexafluoride) and the streamer mode (Argon, Freon, Isobutane).

The quality of the mixed gas inside the RPC determines the performance of the RPC. The contaminants like air, moisture, breakdown radicals of gas like HF, CF3 etc. lead to the deterioration of the detector lifetime and it performance [3][4]. These contaminants are produced due to ionisation process or through leaks in the RPC or the tubes joints connecting the RPCs. To remove the contaminants fresh gas need to flushed into the RPCs and the frequency or the number of volume changes plays a crucial role in the reducing the cost incurred also on the environment impact, due large number of detectors and especially in a project that is envisaged to last for a couple of decades[5][6][7]. 


\subsection{RPC:}

The Resistive Plate Chamber, (RPC) [FIGURE 1A] is an ionization particle physics detector. The performance parameters are Detection efficiency (97-98\%), Time resolution (1.5 to 2 nanosecond), spatial resolution (1 centimetre), low leakage currents (few hundred nanoampheres), low noise rates (few hundred hertz) and long term stability. The elementary component of a RPC is a gap, a gas volume enclosed between two resistive plates (electrodes). The large volume of the total RPC system and the use of a rather expensive gas mixture impose the necessity to use a gas recirculation system.

A schematic diagram of the glass RPC gap that would be used in the final INO-ICAL is as shown in the FIGURE 1B. And the details of the components used in the fabrication of an RPC, are shown in FIGURE 2.

FIGURE 1A : RPC Schematic

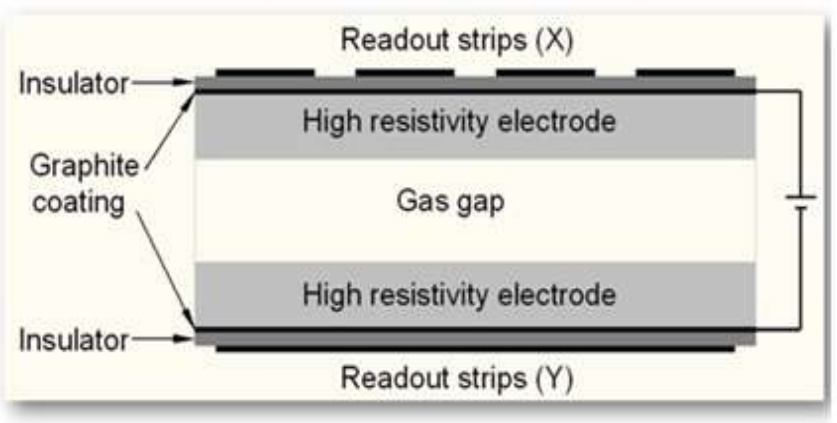

FIGURE 1B: RPC- Electrode (3mm thick glass)

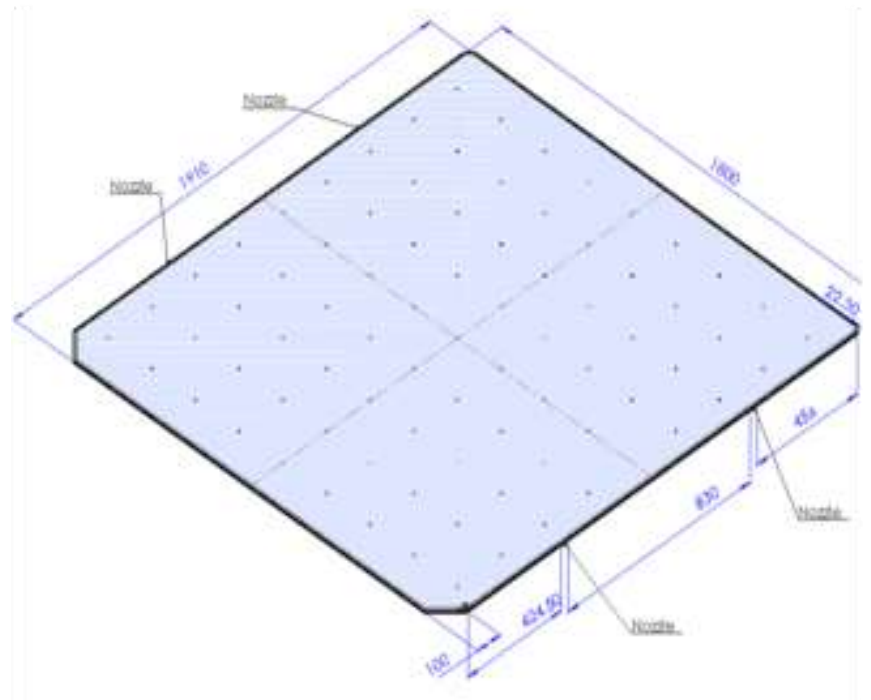

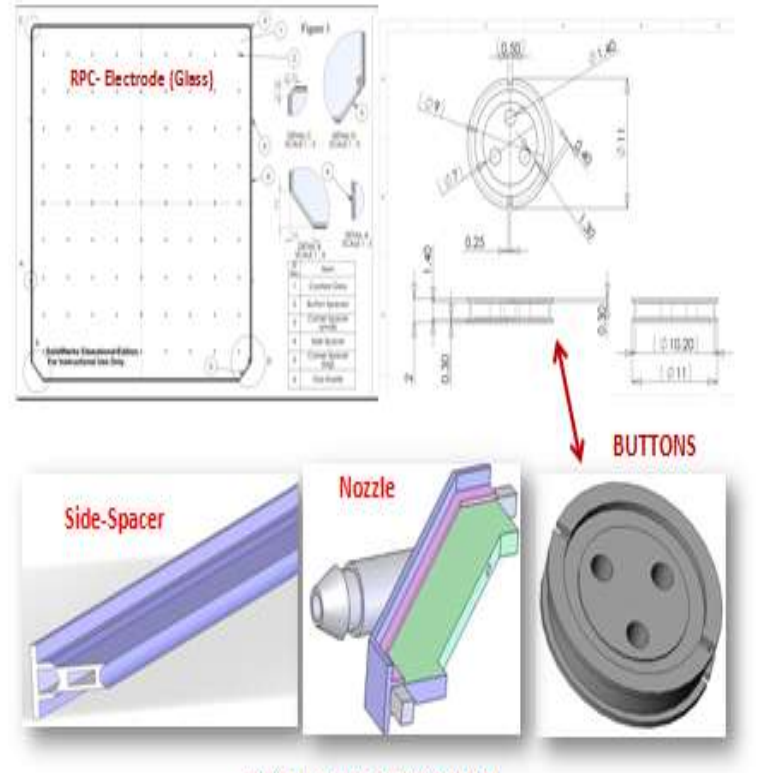

FIGURE2:RPC COMPONENTS

\section{SOFTWARE TOOLS AND CRITERIA:}

The main objective is to conduct a simulation study and understand the gas flow patterns inside an RPC chamber at low velocities. The basic tools for various numerical analyses that are commercially available for fluid analysis are COMSOL, Fluent, Solid works etc. The COMSOL platform is used in this analysis as this will give some cross verification for the simulation work done earlier using Solid works and to decide for further details studies if it can be used.

The gas mixture is stable and chemically inactive but, when exposed to radiation and electrical field, free molecules and or radicals are generated. The ideal conditions for RPC operation would be to keep the supplied gas free of any contaminant of both external and produced in citu during the usage.

\subsubsection{Hardware Details and Software Assumptions:}

The basic components of an RPC gas gap are the float glass of $3 \mathrm{~mm}$ thick, poly carbonate buttons of $2 \mathrm{~mm}$ thick and of diameter $(11 \mathrm{~mm})$, the $\mathrm{T}$-shaped side spacers and gas nozzles FIGURE 2. The geometry has been simplified. The float glass wall is assumed to be adiabatic and having roughness of 0.05 micrometer. The buttons that are placed inside the chamber at $200 \mathrm{~mm}$ spacing are considered to be cylindrical of the exact height of $2 \mathrm{~mm}$. The heights of these buttons play, a crucial role in maintaining the gap or thickness throughout the area of RPC gap, so that the applied field is uniform. The side spacers used are of T-type as shown in FIGURE 2, these side spacers help in holding the glass and act as sealants, so that the Glass gap so formed has leak proof sealing, but since the rectangular section of $\mathrm{T}$-side spacer comes in the contact of gas, during modelling the spacers are considered to be rectangle. The 
gas nozzles are placed at the appropriate location at the entry and exit of gases. These are four in numbers and in lieu of full nozzles, the input holes are considered as two dimensional entry points having nozzle end dimensions $(1.81 \mathrm{~mm})$. The entry point position and the orientation are identical to that of nozzle FIGURE 3.

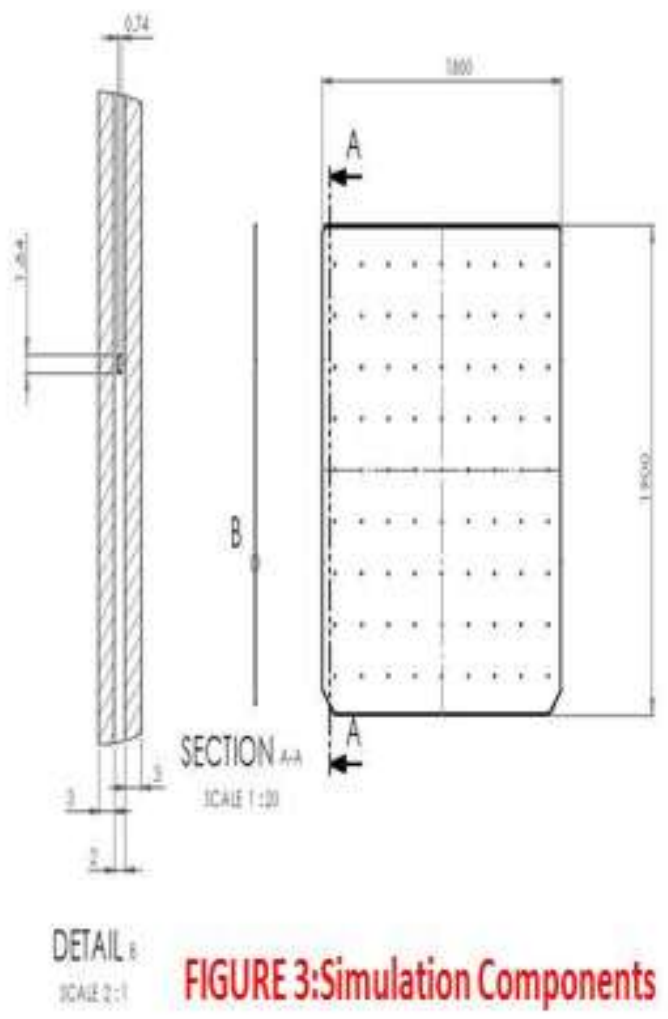

The gas used in the simulation is R22 (assuming that the flow pattern would be similar to R134a) as it is available in the library of COMSOL package. The major concentration of gas mixture is Freon which is $95 \%$ and hence it would replicate as a single gas for simulation purpose. The boundary conditions used are the flow rate at the input taking into consideration the volume changes that would take place for an RPC. As the laboratory is maintained at a fixed temperate and pressure The earlier modules generated in Solid works platform are imported in the COMSOL and the simulation is done in $2 \mathrm{D}$ and it is assumed that the flow is symmetrical about the mid-plane in Y-directions i.e. thickness and taking into consideration.

\subsubsection{Performance- parameter:}

The desired performance of an RPC are (a) Detection efficiency not less than $97-98 \%$ (b) Time resolution around or less than $2 \mathrm{~ns}$ and spatial resolution of less than $1 \mathrm{~cm}$ and the low leakage currents of few nano-amperes. (c) Long term stability over a period of decade. (d) The breakdown radicals are invariably produced during operation of RPC. The residence time of the radicals within a gap is related to the drift and diffusion properties. The least residence time for radicals is desirable for extended life span of a RPC.

\subsubsection{Physics perspective:}

The Freon 134a acts as a main Ionisation gas. The Isobutane gas helps in absorbing the secondary UV photons and SF6 helps in absorbing electrons and stops the streamer formation. The quencher (iC4H10) and electronegative (SF6) gases will help in containing the charge development and reducing the streamer probability, the more Isobutene better is the performance, but more than $8 \%$ is not permitted due safety issue. If the percentage of SF6 is increased, greater than $0.3 \%$, the noise rate gets reduced, efficient is also reduced and deteriorate the time resolution of the RPC operation. If Freon concentration is reduced the efficiency and noise rate is reduced and hence timing gets affected. Therefore the appropriate concentration of gases is to be maintained.

Therefore it is mandatory that the air must completely be displaced by the gas mixture in the entire gap of the RPC detector. The concentration of Isobutene should not vary by few percent5. The concentration of SF6 should not vary more then $1 \%$.

The optimised ratios of the gas mixture are 95.0:: 4.5: 0.5 to get the best performance of the RPC. The gas mixture ratio need to maintained uniform throughout the volume of the RPC detector. If any deviation is created within localised area such as pockets then the performance would be affected in localised or overall area of RPC.

\section{INTERPRETATION OF GRAPHS.}

Part A: The Part A of simulation, consists of plots showing Flow lines called 'Isoflow'. An Iso -flow is defined as a line joining points of equal magnitude of gas velocities in a 2D plane or 3D space, bunched to form a particular range flow velocity. A single component gas flow is introduced at time $\mathrm{t}=0$ into the gap. The subsequent development of Isoflow lines through different part of the gap after a period starting from $=5.45 \mathrm{sec}$. to $\mathrm{t}=60 \mathrm{sec}$ are reported here.

The distribution of gases after introduction through inlet nozzle is under study. This is important from many points such as how each component of gas mixture is going to develop its partial pressure over a period of time from inlet to the detector to the outlet of the detector.

This study is also important for estimating extent and movement of a radical cluster which might form at time $\mathrm{t}=0$ at any point inside the RPC and then travel towards inlet or outlet. Since the motion of gas molecules is in Laminar range the partial pressure of radical, a point source, will be some form of mathematical such as Gaussian distribution by combining diffusion and drift under pressure gradient. This model can also be applied to the inward leakage of impurity from air such as moisture and oxygen and the distribution as if from an infinite source. For flow rate of (SCCM) ,the Isoflow lines develop stronger in the middle and less at edge initially. The steady state condition is achieved before 60 sec. 


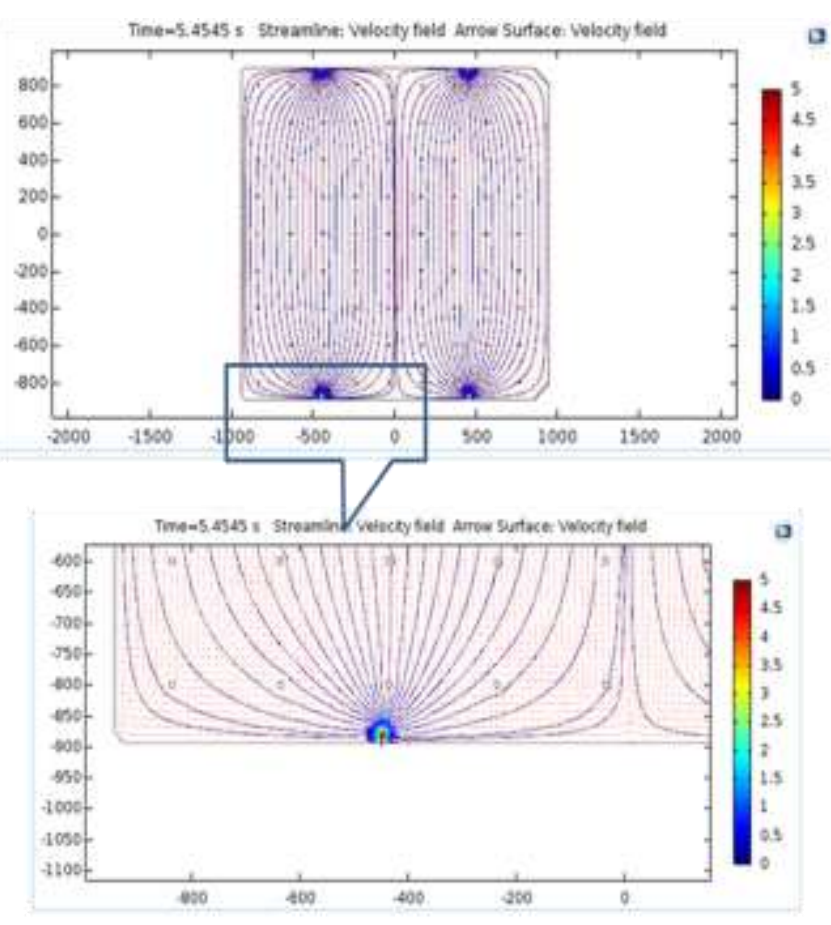

FIGURE :4A :Transient Flow @5.45 seconds [bottontigure shows the blowupl
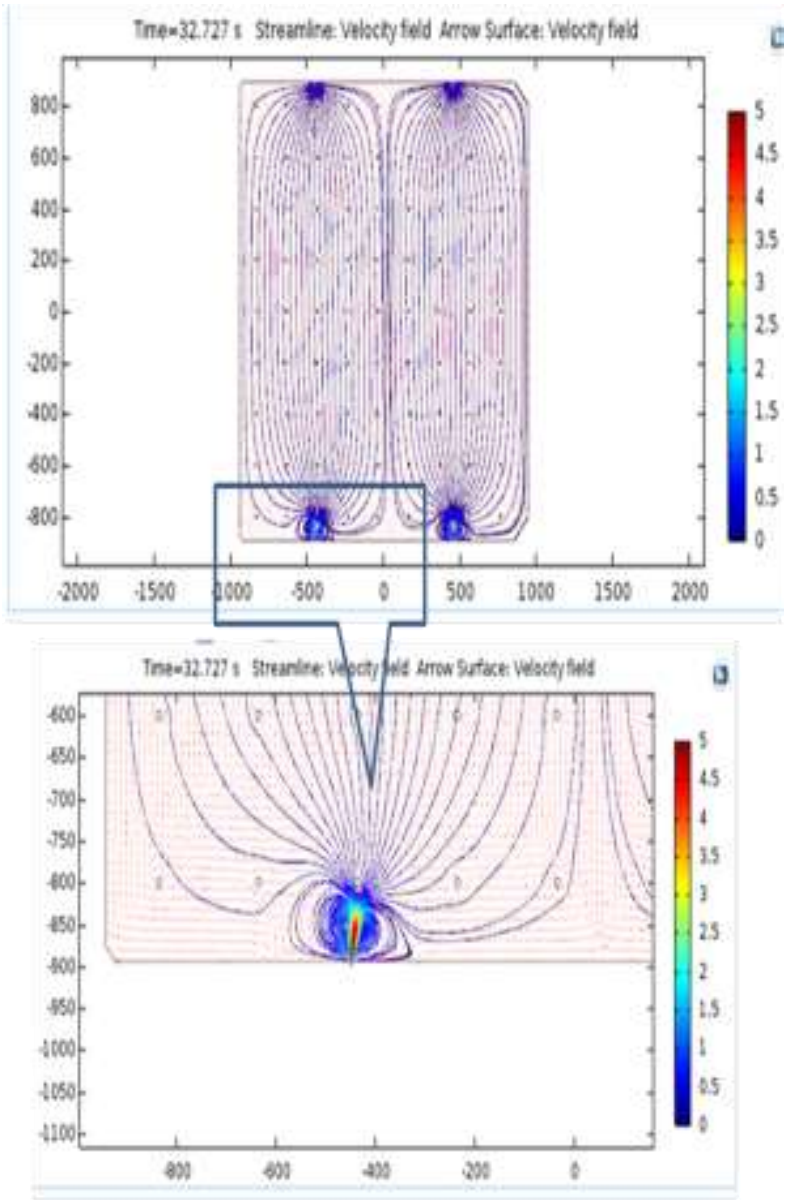

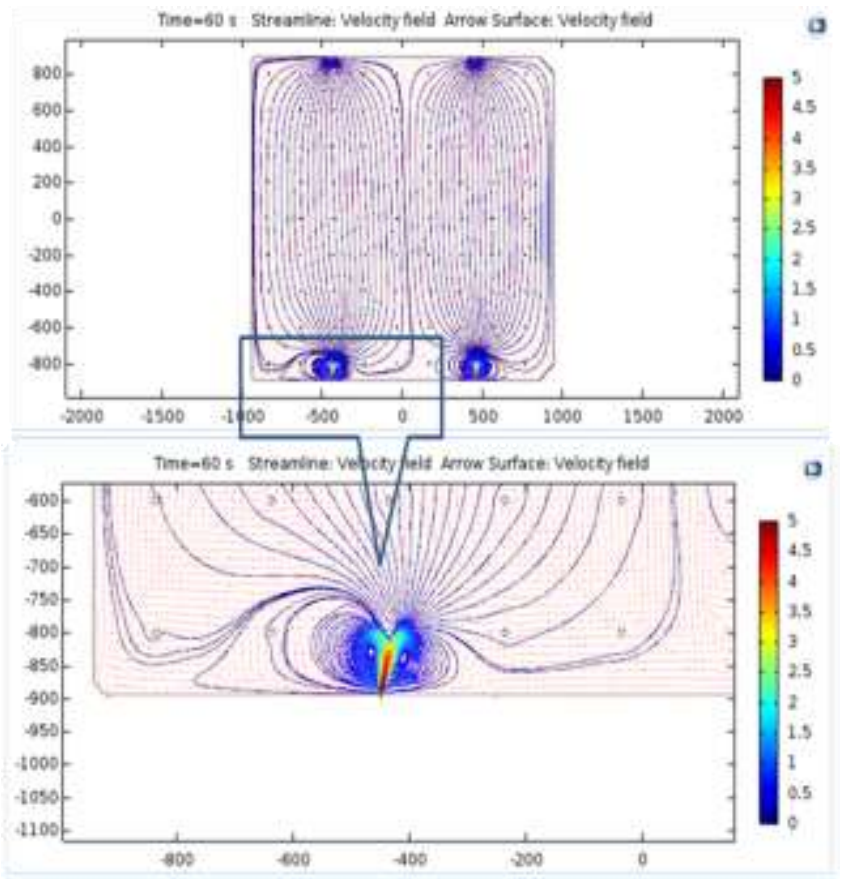

FIGURE :4C :Transient Flow @60 seconds (Bontem tieure shows the blowup)

Part B: In the Part B simulation i.e. the second set of simulations the "Isoflow" distribution is studied after steady state conditions have been reached. The plots in FIGURE5A, FIGURE 5B and FUGURE 5C represent the simulation reports. The "isoflow" distribution is studied for different velocities starting from 0.02 meters/second $(1.51$ $\mathrm{SCCM}$ ) to 0.17 meters/second (12.852), while range of interest of operation for a single glass RPC is about 6 SCCM.

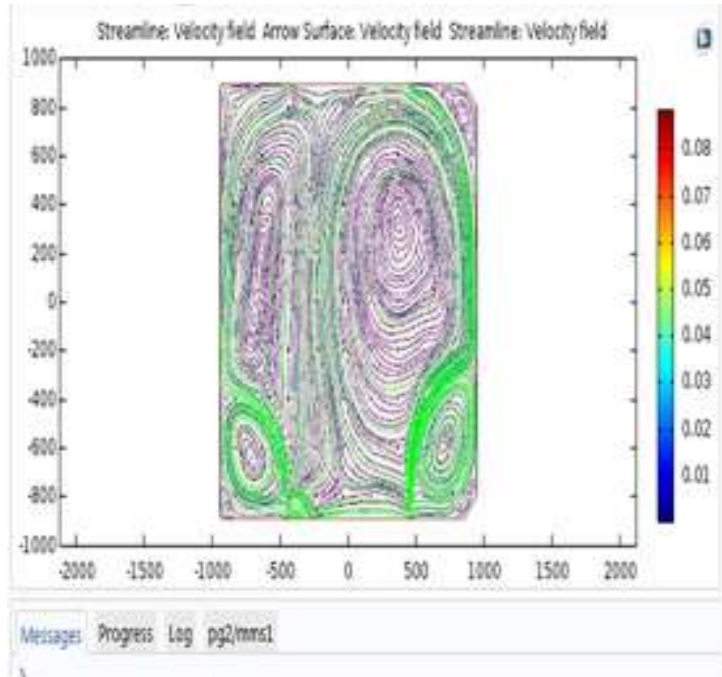

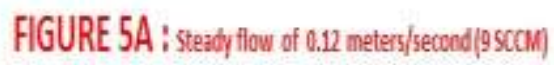

FIGURE:AB :Transient Flow @32.7 seconds

[eotton figure shous the blow.p] 


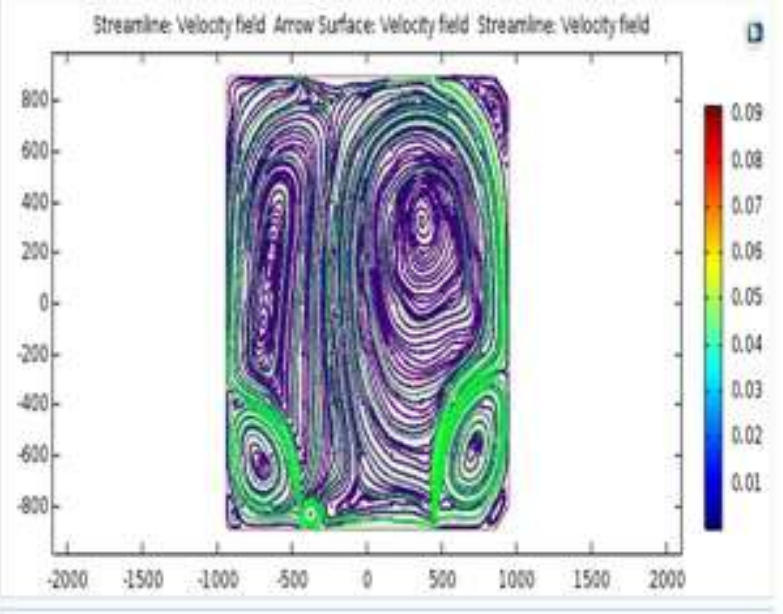

FIGURE $5 B$; steady flow of 0.08 meters/second (6scam)

Part C: In the Part $\mathrm{C}$ simulation plots, the surface height velocity magnitude plots (Figure $5 \mathrm{~A}$ and Figure $5 \mathrm{~B}$ are presented. The plots in Figure 5C and Figure 6D. There are two inputs gas nozzles and two output nozzles and it looks that the flow of gas reaching at both the output nozzles is different [FIGURE 6A, FIGURE 6B and FIGURE 6C] and hence and experimental setup is done to confirm, if the flow of gas is different at the two outputs.

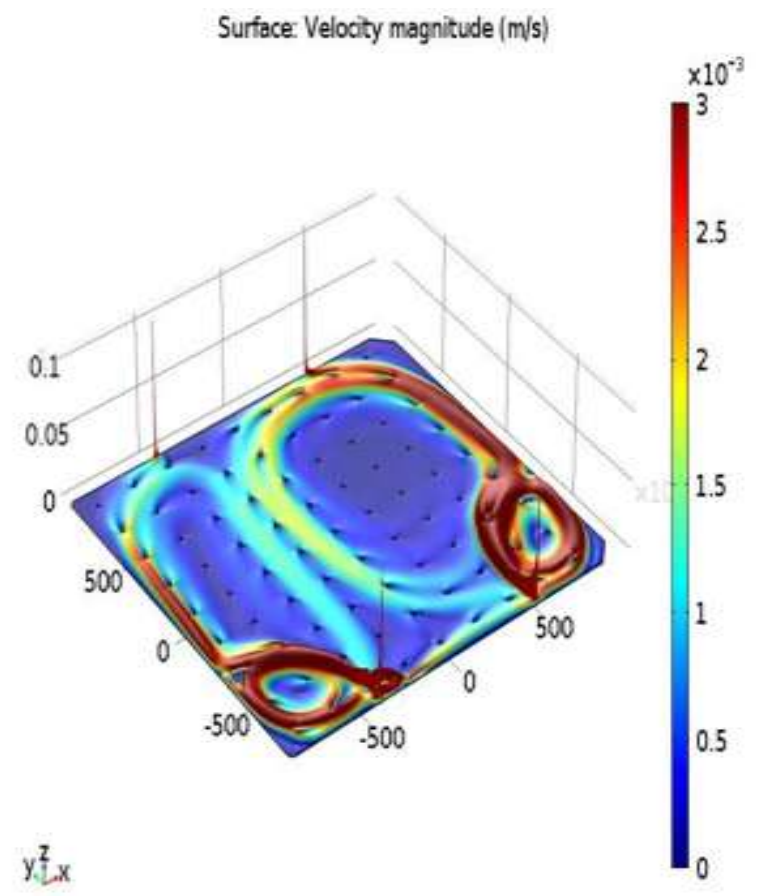

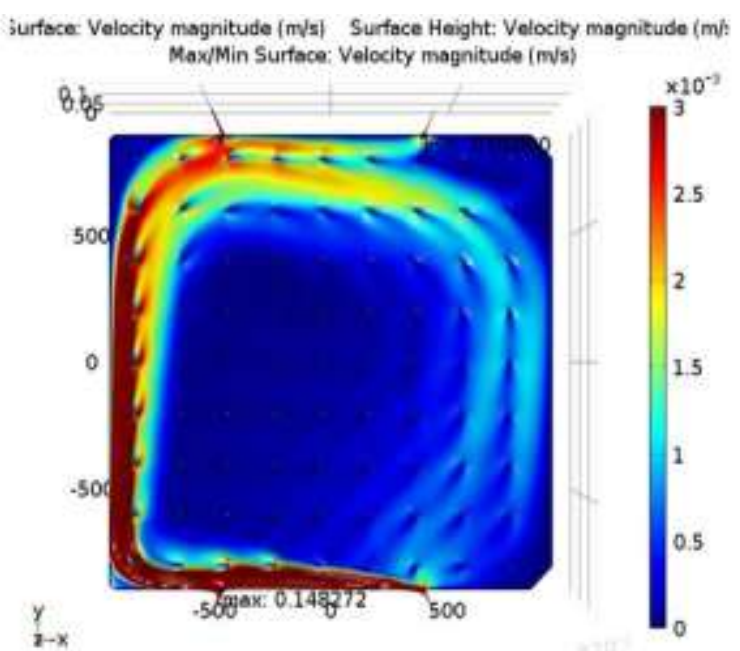

FIGURE 68 : Volocity magnitude plot (Flew not uniform in beth output)

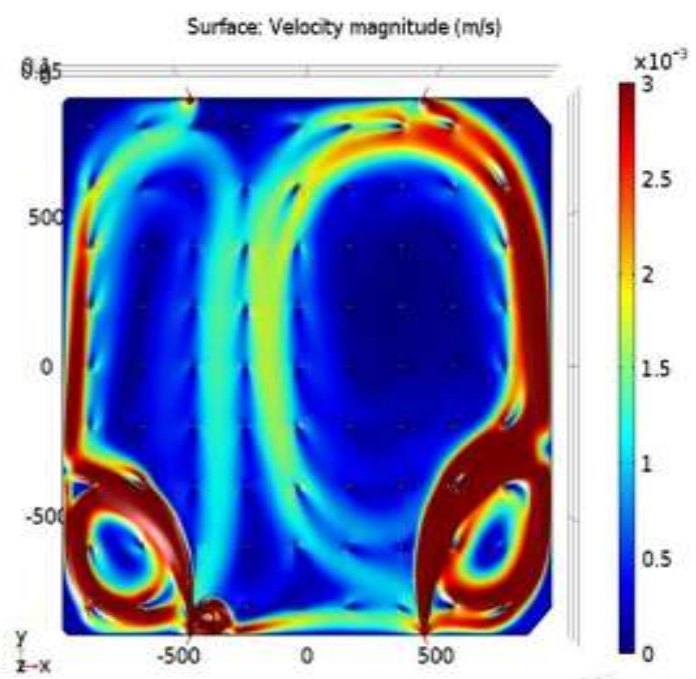

FIGURE 6C : Velocitymagnitude plot(Flow not uniform in both output]

\section{EXPERIMENTAL SETUP :}

To verify the simulation result (velocity magnitude plot) that the flow of gas is more in one of the outputs of the RPC gaps:

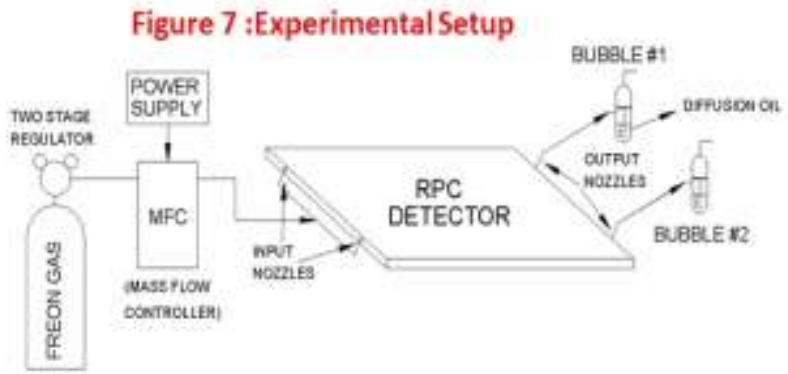

FIGURE 6A : Velocity magnitude plot (Flow not uniform in both output) 
A one meter by one meter RPC with two inputs and two outputs is connected as shown the above Figure 7 . The Freon (R134a) gas is used and the RPC under test is connected as shown above. The MFC supplies gas as per the required setting of gas flow from few SCCM to tens of SCCM s to the RPC under test. The gas flows through the detector and then moves into the bubblers connected at the output. The length(s) of each tubes going to the input and output of the RPC used are of equal lengths to make sure that equal quantity of gas is flown. The number of bubble's are counted in each of the bubbler. It is observed the numbers of counts of bubbles in each bubbler are SAME and moreover the bubbler counts remain same even when the bubbler connections are interchanged. Hence the simulation flow patter seen does not match with experimental setup may be because of the inadequate and precise measuring tool that may be needed to match the simulation results and also the flow may be unequal in the initial stage which we are not able to count. More detailed study may be required.

\section{CONCLUSION:}

\section{Regarding The Software Tool Used:}

So far we have used two packages viz. Solidworks simulation package and COMSOL Multiphysics ${ }^{\circledR}$ package. The results are presented in this paper are using the COMSOL package and is the learning curve for us. The basic tools or modules generated in the solid works platform are imported in the COMSOL and simulation is done in 2D and could not perform in $3 \mathrm{D}$ due to the non availability of high end PC or a server.

\section{RESULTS:}

[1]. The basic and preliminary results presented are primitive and are done to check if the COMSOL software package could be used for detailed gas flow and control patterns inside the RPC gaps compared to the Solid works (used earlier), but looks the COMSOL package is more complex in nature, than the solid works for the beginners.

[2]. The result indicates that, the transient and steady flow of gas could be easily done in the COMSOL package.

[3]. As the motion of gas molecules is found to be in Laminar range, the partial pressure of gas component or radical, (a point source to form Gaussian distribution) can be calculated at any instance by combining diffusion and drift under small pressure gradient. It is also possible to apply the model to the inward leakage of impurity (infinite source).

[4]. The verification using an experimental setup needs more precision of measurement for the low flow rate, as the simulation gives a high degree of precision and hence could not verified that there is a possibility of different flow rates at the output of the RPC detector.

\subsection{Future Work :}

The main goal is to have a working model for the complete flow and control of mixed gas into a RPC and latter to have connected multiple (four) RPC detectors in a daisy chain. Using the Solidworks platform may be a better choice for our study for the flow patter with higher values. Eventually we need to achieve the results comparable with CERN CDF team [10]. And then conclude on "flow and control of gas mixture" for the RPC's.

The physics perspectives are yet to be achieved.

\section{ACKNOWLEDGMENTS:}

The research students of applied electronics department of Gulbarga University have helped in building few modules in simulation process etc.

\section{REFERENCES:}

[1]. INO Collaboration, INO Project Report, Technical Report INO/2006/01, 2006; Detailed Project Report I, Technical Report, 2007; Detailed Project Report on INO-ICAL Detector Structure II, Technical Report, 2008

(http://www.ino.tifr.res.in/ino//OpenReports/INORep ort.pdf).

[2]. M.Bhuyana et al, Development of glass Resistive Plate Chambers for INO experiment, NIM A 602 (2009) 744-748.

[3]. M.Bhuyana,V.M.Datarb.et al, Development of $2 \mathrm{~m} \times$ $2 \mathrm{~m}$ size glass RPCs for INO

NIMA_PROCEEDINGS-S-10-00275-1.fdf.pdf.

[4]. M.R.Bhuyan et al, Preliminary results on optimization of gas flow rate for ICAL RPCs: Nuclear Instruments and Methods in Physics Research A 736(2014) 135-142

[5]. M.Bhuyana et al, Performance of the Prototype Gas Recirculation System with built-in RGA for INO RPC system. doi:10.1016/j.nima.2010.09.169

[6]. Abbaneo D. et al, Study of gas purifiers for the CMS RPC detector. Nuclear Instruments \& Methods In Physics Research. Section A, Accelerators, Spectrometers, and Detectors And Associated Equipment, vol. 661, p. S241-S244, ISSN: 01689002, DOI: 10.1016/j.nima.2010.08.089.

[7]. R.Guida et al, Optimization of a closed-loop gas system for the operation of Resistive Plate Chambers at the Large Hadron Collider experiments. Nuclear Instruments and Methods in Physics Research A 661 (2012) S214-S221

[8]. M. Bosteels et al, CMS Gas System Proposal, CMS Internal Note CMS IN 1999/018 (1999)

[9]. M. Capeans et al, RPC performances and gas quality in a closed loop gas system for the new purifier's configuration at LHC experiments. JINST: Special Issue On Resistive Plate Chambers And Related Detectors Rpc2012.

[10]. CERN CDF-team: Jun 30th 2009 by Waldemar Maciocha Antonio Romanazzi. 